\title{
Procesamiento de biopsias de piel en el laboratorio de patología
}

\author{
Wilber Javier Martínez', Rodrigo Restrepo² \\ 1. Médico patólogo, especialista en Dermatopatología y en Gerencia de IPS, profesor de Dermatopatología Universidad Autónoma de Bucaramanga, \\ Bucaramanga, Colombia. \\ 2. Médico dermatopatólogo, jefe programa de especialización en Dermatopatología Universidad CES, profesor de Dermatopatología Universidad \\ Pontificia Bolivariana, Medellín, Colombia.
}

\section{Resumen}

El procesamiento de las biopsias de piel es el primer paso en el que interviene el dermatopatólogo. Este importante evento es a menudo delegado en personal escasamente entrenado, como auxiliares o estudiantes de posgrado en sus primeros años de formación. Las consecuencias de esta acción son generalmente irreversibles y habitualmente los errores cometidos no pueden repararse.

PALABRAS CLAVE: biopsia, laboratorio, dermatopatología, márgenes de resección.

\section{Summary}

The processing of skin biopsies is the first event in which the dermatopathologist is involved. This important step is often delegated to poorly trained staff including support staff or undergraduated students in their formative years. The consequences of this action are generally irreversible and usually the mistakes cannot be repaired.

KEYWORDS: biopsy, laboratory, dermatopathology, resection margins.

\section{Correspondencia:}

Wilber Javier Martínez

Email:

wilberjmc@hotmail.com

Recibido: 30 de diciembre de 2013. Aceptado: 12 de febrero de 2014.

No se reportan conflictos de interés.

\section{Introducción}

En este artículo se revisan las técnicas y métodos más apropiados para el procesamiento de material quirúrgico relacionado con lesiones de piel, cuero cabelludo, uñas y bordes mucocutáneos, con el fin de suministrar una guía de referencia para el manejo de las biopsias recibidas en los laboratorios de patología y dermatopatología. El adecuado procesamiento de una biopsia es el primer paso que permite bridarle al médico tratante un reporte con la información necesaria para tomar decisiones terapéuticas acertadas y definir mejor el pronóstico de los pacientes.

\section{Conceptos generales}

Hay un vacío en el conocimiento concerniente al procesamiento del material quirúrgico relacionado con la piel. La mayoría de los laboratorios de patología no tienen procedimientos adecuadamente estandarizados que tengan en cuenta las diferentes variables que afectan dicho proceso.

En primer lugar, el dermatólogo debe tener conocimientos básicos de dermatopatología que le permitan saber cómo solicitar un estudio de patología y cómo interpretarlo. A su vez, el patólogo debe tener suficiente entrenamiento en dermatopatología que le permita manipular adecuadamente los tejidos y proponer un diagnóstico o diagnósticos diferenciales relevantes, según el contexto clínico ${ }^{1}$. El tipo de técnica de biopsia escogida, ya sea por escisión (completa) o por incisión (parcial) es una decisión del dermatólogo, basada en su conocimiento de los diagnósticos diferenciales por considerar en un paciente en particular, además de la localización de la lesión y su tamaño . Una biopsia parcial en sacabocados o por afeitado puede conducir a un mal diag- 

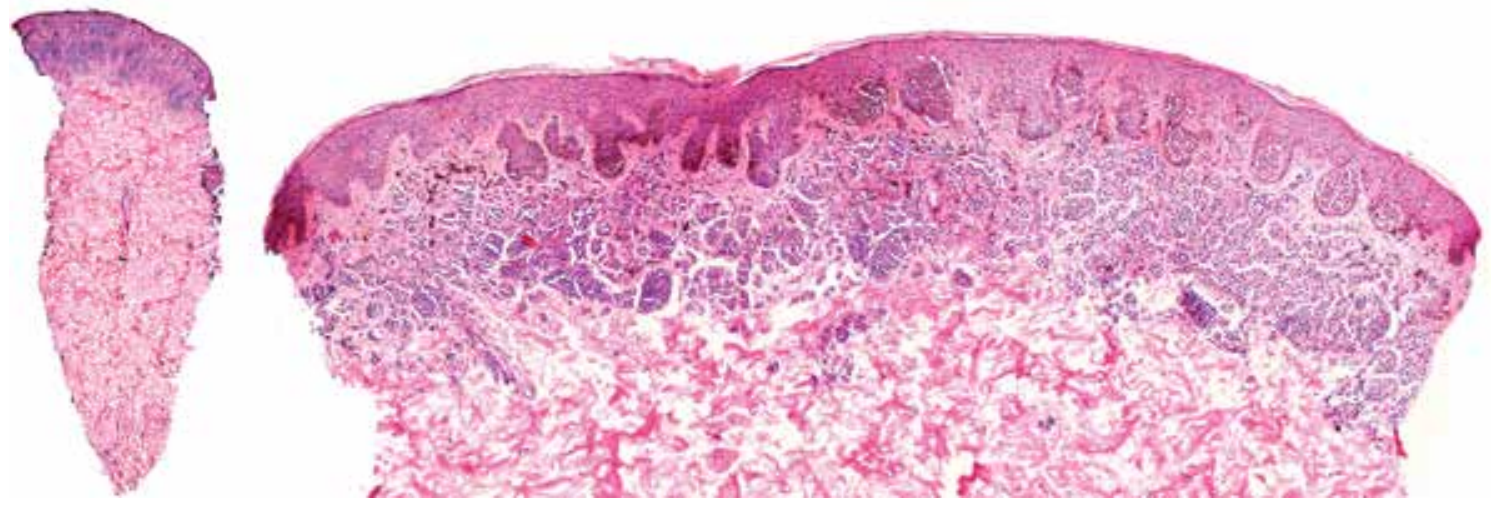

FIGURA 1. Biopsia de un nevus. A la izquierda se observa una biopsia en sacabocado de $3 \mathrm{~mm}$ de diámetro que no incluye toda la lesión. Hematoxilina y eosina, 20X. A la derecha se observa la misma lesión a mayor aumento. No es posible analizar en los bordes si hay extensión o no del componente epidérmico sobre el dérmico para confirmar o descartar un nevus displásico. Hematoxilina y eosina, 200X.
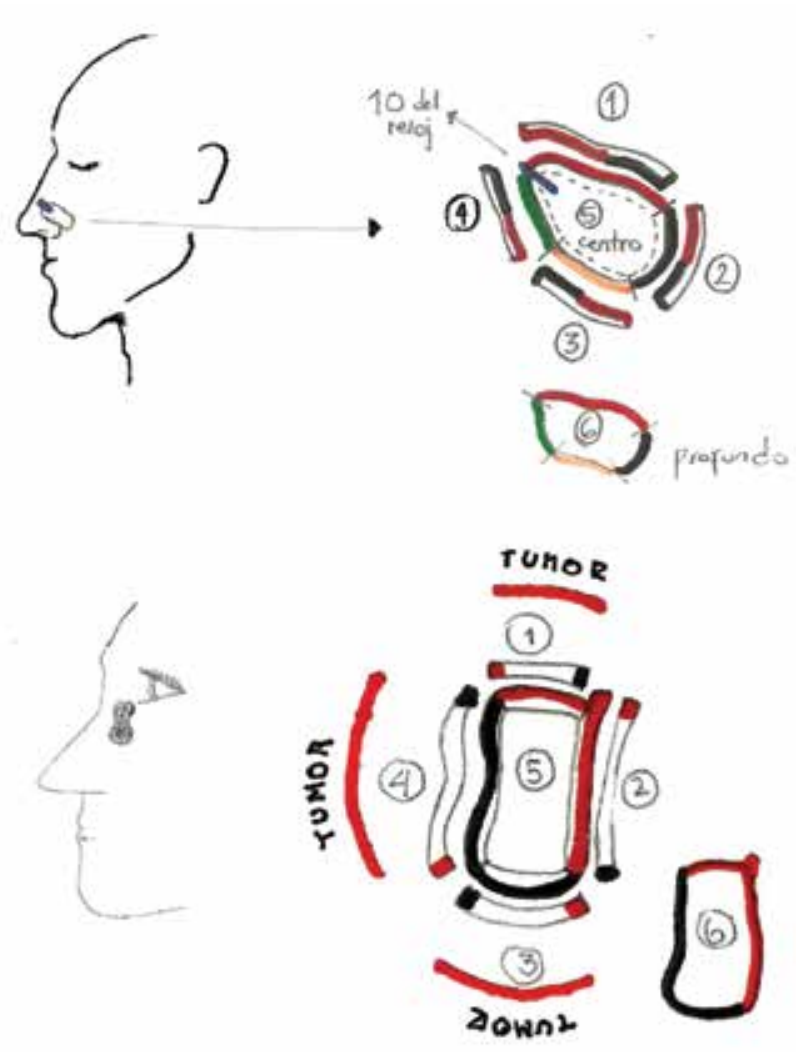

FIGURA 2. Esquemas de dos pacientes con carcinoma basocelular del ala y la raíz nasal. Los esquemas permiten identificar en un solo diagrama la localización de la lesión, la orientación espacial de los cortes en relación con el paciente y los códigos de colores empleados para identificar microscópicamente cada borde como superior, inferior, interno y externo. nóstico histopatológico debido a un muestreo no representativo; dichos errores pueden ocurrir entre el $16 \%$ y el $43 \%$ de las biopsias parciales ${ }^{3}$. Dos situaciones en las que puede haber limitaciones en la información que proveen las biopsias preoperatorias por incisión son:

- Cuando con la biopsia se demuestra claramente una enfermedad, como un carcinoma in situ y, simultáneamente, hay focos de invasión en otras porciones no representadas en la misma, como puede suceder en un afeitado de un lentigo maligno.

- Cuando una biopsia parcial no incluye la arquitectura global de una neoplasia, lo que impide su análisis correcto, como por ejemplo sucede en el diagnóstico diferencial entre carcinoma escamocelular invasor y un queratoacantoma, o entre un melanoma y una lesión melanocítica benigna, o entre esta y un nevus displásico (FIGURA 1).

En estos casos el patólogo debe resaltar dichas limitaciones en su reporte final y, en la medida de lo posible, dejar claro que estas imprecisiones en el diagnóstico se pueden minimizar al obtener una biopsia más amplia ${ }^{3,4}$.

En relación con las biopsias producto de la extirpación quirúrgica de un tumor, las fotografías o diagramas de los especímenes macroscópicos recibidos pueden ser definitivos en asegurar el óptimo muestreo del espécimen, permitiendo a los dermatopatólogos depender menos de los juicios y descripciones escritas de quien hace la disección del espécimen en las salas de procesamiento macroscópico (FIGURA 2) 5 .

Otro elemento importante que se debe tener en cuenta es que los reportes de patología de las resecciones de piel de las lesiones tumorales describen márgenes que son significativamente menores de los planeados para 

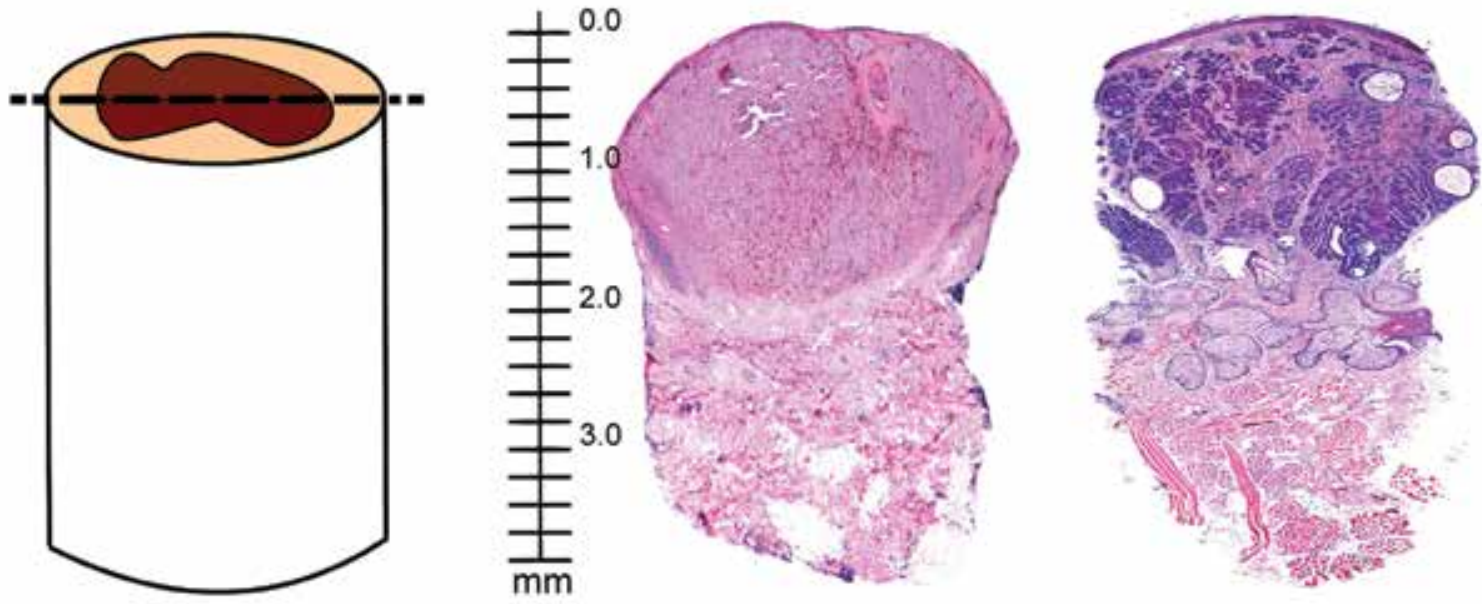

FIGURA 3. Biopsia con sacabocado. A la izquierda, el plano de sección es de adentro hacia afuera; en el centro, se observa un melanoma con índice de Breslow de 2, y a la derecha, un carcinoma basocelular con infiltración similar. En el caso del melanoma, la lesión está totalmente representada y el índice de Breslow es definitivo. En el caso del carcinoma, los márgenes laterales están comprometidos, por lo que el cálculo de la profundidad de la infiltración es únicamente tentativo, al no disponerse de todo el espécimen. Hematoxilina y eosina, $20 X$.

la escisión quirúrgica. Los factores que causan tal hallazgo son:

- La extensión lateral no detectada clínicamente de un tumor.

- El encogimiento de la piel después de la resección.

Esta última variable se debe fundamentalmente a la contractilidad intrínseca del tejido ${ }^{6}$. En promedio, los tejidos se encogen el $21 \%$ en longitud y $12 \%$ en anchura. De forma general, se ha evidenciado que los especímenes se encogen de manera global entre el $14 \% \mathrm{y}$ el $16 \%$ después de la resección y el procesamiento histológico ${ }^{6,7}$. El incremento en la edad y la elastosis solar se correlacionan con menor encogimiento. Por otra parte, la reducción de tamaño según el sitio anatómico es variable, siendo $5 \%$ mayor sobre el tronco comparado con la región de la cabeza y el cuello.

Como dato significativo, la fijación en formol no es la causa del encogimiento del tejido; por el contrario, conlleva una ligera expansión, según lo estudiado ${ }^{7}$. Además, se han observado diferencias entre la piel que contiene un tumor, la cual se encoge $11 \%$, en promedio, y la piel adyacente libre de tumor en el mismo plano, la cual lo hace $19 \%$, en promedio, lo que muestra que el encogimiento no es uniforme en todo el espécimen ${ }^{6}$. No se debe olvidar que un corte oblicuo de un material mal incluido, puede producir falsas interpretaciones en relación con la amplitud de los márgenes de resección ${ }^{8}$.

\section{Biopsias con sacabocados}

Es una de las formas más frecuentes en que los dermatólogos toman muestras para estudio histopatológico. Aunque con este tipo de biopsia no se pueden evaluar exhaustivamente los márgenes de resección, son apropiadas para hacer diagnósticos histológicos de rutina. Se toman en diferentes diámetros y cuando miden 4,0 mm o más deben cortarse verticalmente en dos secciones $^{9,10}$. Siempre se debe tratar de extirpar toda la lesión, particularmente si es importante tomar algún tipo de medida numérica o cualitativa de la cual dependa la planeación del procedimiento quirúrgico adecuado (FIGURA 3).

Como toda biopsia de piel, su pequeño tamaño la hace muy propensa a presentar artefactos por aplastamiento con las pinzas al tratar de separarla de la dermis, y por torsión, cuando se usan sacabocados reutilizados que no cortan adecuadamente. Igualmente, son muy propensas a la desecación y electrocoagulación, por lo que no deben dejarse al aire libre y siempre se debe utilizar un bisturí sin electrocauterio asociado (FIGURA 4).

En este tipo de biopsias generalmente no se reportan márgenes, ya que siendo este un espécimen cilíndrico, el plano del corte siempre se selecciona al azar debido al infinito número de posibles planos tangenciales que se podrían escoger (FIGURA 5) ${ }^{9}$. Realmente, en un espécimen obtenido mediante sacabocado el único margen $100 \%$ confiable de reportar es el positivo ${ }^{11}$. 

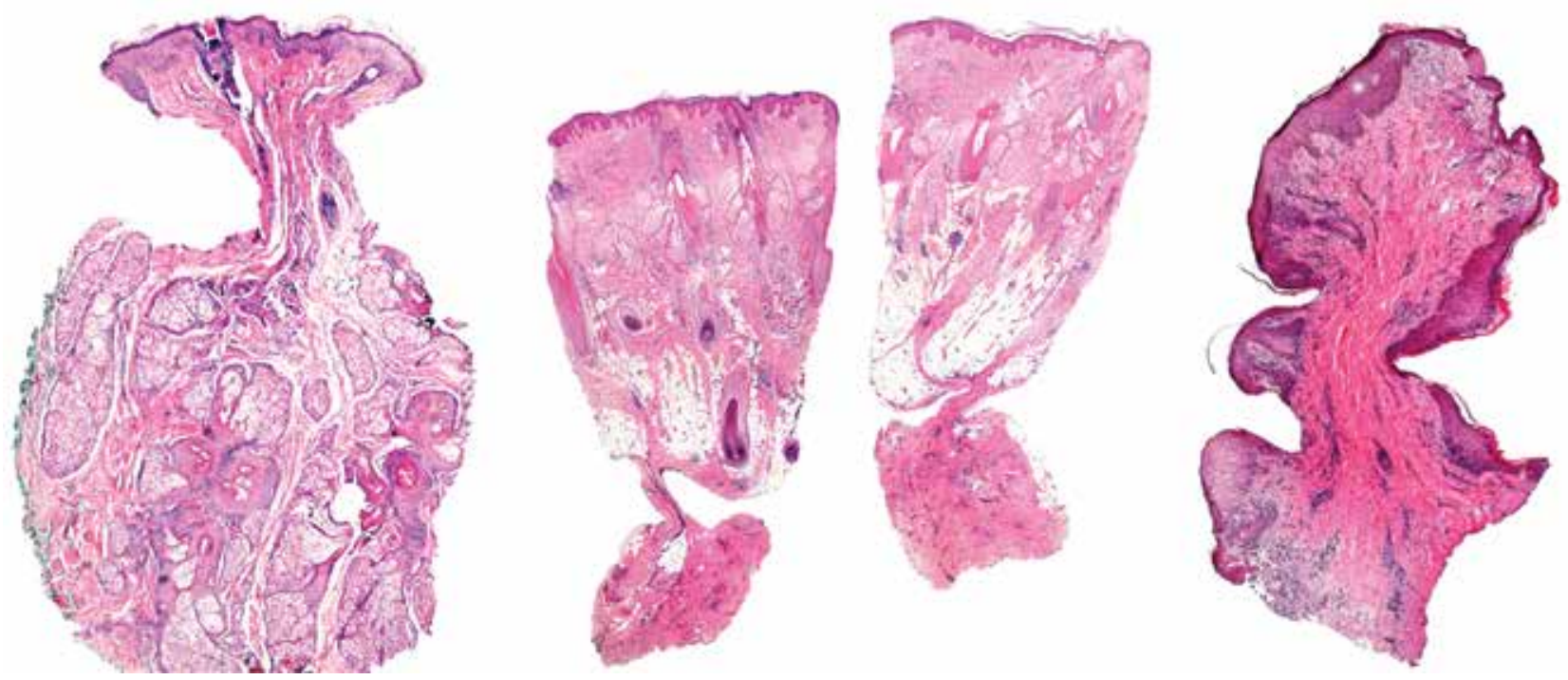

FIGURA 4. A la izquierda y en el centro, biopsias con sacabocados muy dañadas por aplastamiento debido al uso inadecuado de las pinzas, tanto en su porción superior como inferior. La biopsia del lado derecho está totalmente deformada y aplastada por el uso inadecuado de una pinza con garra. Hematoxilina y eosina, 2oX.

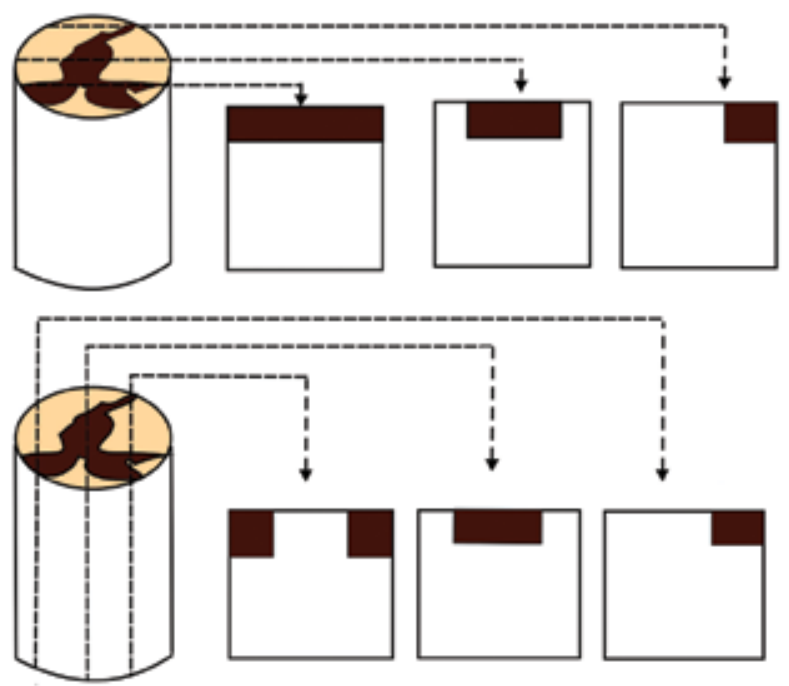

FIGURA 5. Márgenes de resección en biopsia con sacabocados. En este esquema se puede apreciar que, al seleccionar diferentes planos de sección y hacer cortes seriados de un espécimen cilíndrico, los fragmentos obtenidos muestran diferentes puntos de compromiso del margen e, incluso, muchos de ellos no muestran ningún compromiso de los bordes en las secciones microscópicas obtenidas, dando la falsa impresión de bordes libres.

Se ha observado que en las biopsias con sacabocados de lesiones melanocíticas, supuestamente por escisión desde el punto de vista clínico, el $56 \%$ de los especímenes tiene márgenes positivos y el $30 \%$ de las resec- ciones de este tipo, negativas en los cortes histológicos iniciales, presentan márgenes positivos después de hacer cortes seriados en los bloques de manera exhaustiva ${ }^{9,12,13}$. Además, en las lesiones de tipo inflamatorio y neoplásicas se ha encontrado que $12 \%$ de las biopsias con sacabocado no son diagnósticas al usar cortes en una sola lámina y que hasta en $3 \%$ de las que habían tenido un diagnóstico inicial, al hacerles cortes seriados suficientes, el diagnóstico cambia.

El error debido al muestreo incompleto a través del bloque de parafina es una causa reconocida en patología de diagnósticos falsos negativos. Por lo tanto, se recomienda hacer suficientes cortes seriados de la muestra en caso de diagnósticos negativos, o que no coincidan con el cuadro clínico. El inconveniente de esta conducta es que no quede suficiente tejido para estudios complementarios, como los de histoquímica e inmunohistoquímica, por lo cual se debe tratar de no agotar toda la biopsia con cortes aleatorios desde el comienzo ${ }^{13}$. Además, la presencia o ausencia de pigmento, que es clínicamente evidente a la hora del procedimiento quirúrgico, se hace menos evidente después de la fijación en formol y no se puede usar confiablemente como guía por el dermatopatólogo para orientar el espécimen y planificar los cortes 9 .

Otra limitación del estudio de biopsias pequeñas es que un margen comprometido puede perderse involuntariamente cuando se trabaja con el bloque en el micrótomo, al hacer cortes seriados y nivelar el bloque de pa- 


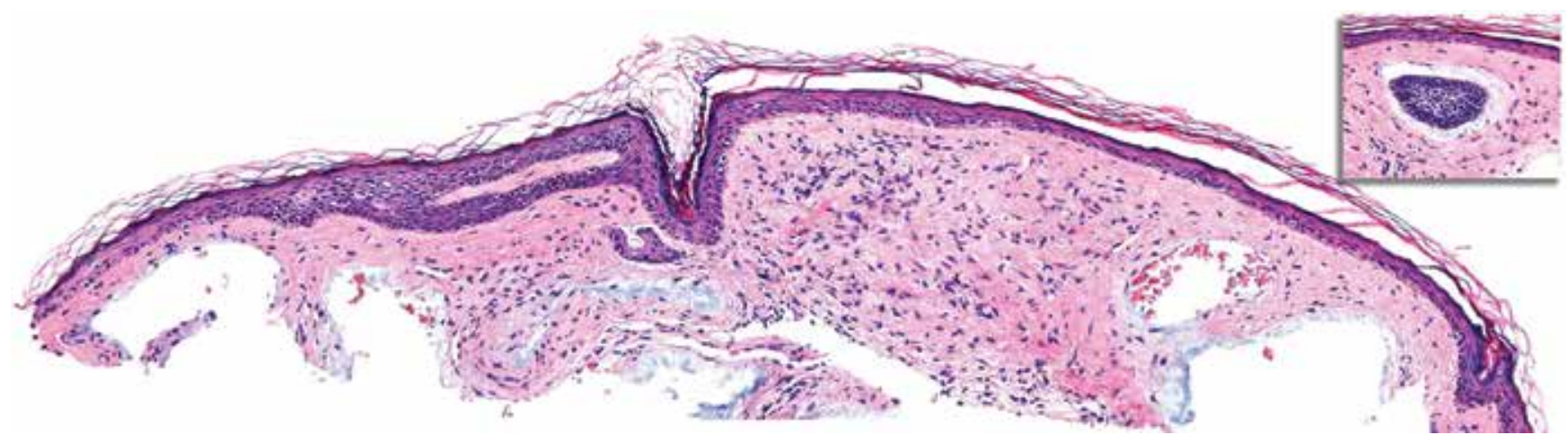

FIGURA 6. Signo del espacio vacío. En este corte todo el carcinoma basocelular ha desaparecido. Solo en los niveles más profundos fue posible detectar la presencia del carcinoma (inserto superior derecho). Hematoxilina y eosina, 40X.

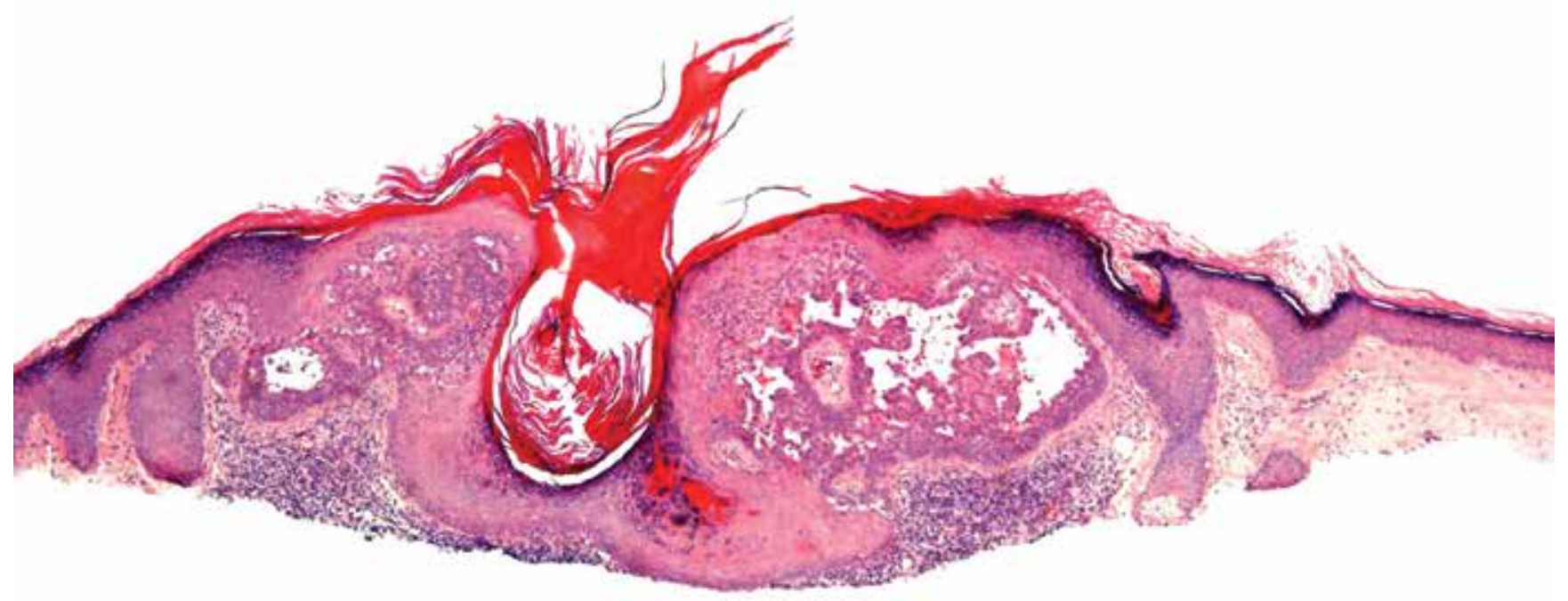

FigURA 7. Saucerización. Las lesiones exofíticas o endofíticas se pueden resecar fácilmente con esta técnica, como en este caso de un carcinoma escamocelular acantolítico con invasión temprana, originado en una queratosis actínica. Hematoxilina y eosina, 40X.

rafina, incluso antes de obtener los fragmentos de tejido que van a quedar en la lámina histológica definitiva9. Ocasionalmente, por razones técnicas, también puede perderse la mayor parte o todo el tumor, particularmente cuando se trabaja con carcinomas basocelulares con mucha retracción y mucina del estroma, quedando únicamente espacios vacíos (FIGURA 6).

En conclusión, y dados los factores antes mencionados, se recomienda no reportar el estado de los márgenes en las biopsias con sacabocados, a no ser que sean positivos. Por lo tanto, si la evaluación de los márgenes es crucial en una lesión, el camino más seguro es hacer una resección en elipse ${ }^{11}$.

\section{Biopsias por afeitado y saucerización}

La biopsia por afeitado es una de las técnicas más comúnmente usadas en dermatología debido a la rapidez con que se puede hacer, la simplicidad del cuidado de la herida y sus muy buenos resultados $\operatorname{cosméticos}{ }^{14}$. Se obtienen discos delgados de tejido que son muy útiles para la evaluación de lesiones muy superficiales, epidérmicas o dérmicas, tal como sucede en el vitíligo, la micosis fungoide en parche, el nevus de unión, los carcinomas basocelulares superficiales, las queratosis 


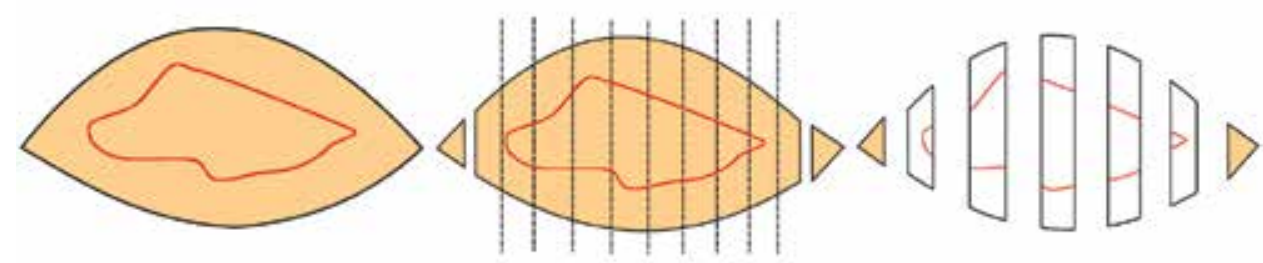

FIGURA 8. Cortes verticales seriados en tajada de pan. En el panel superior se observa la representación esquemática de

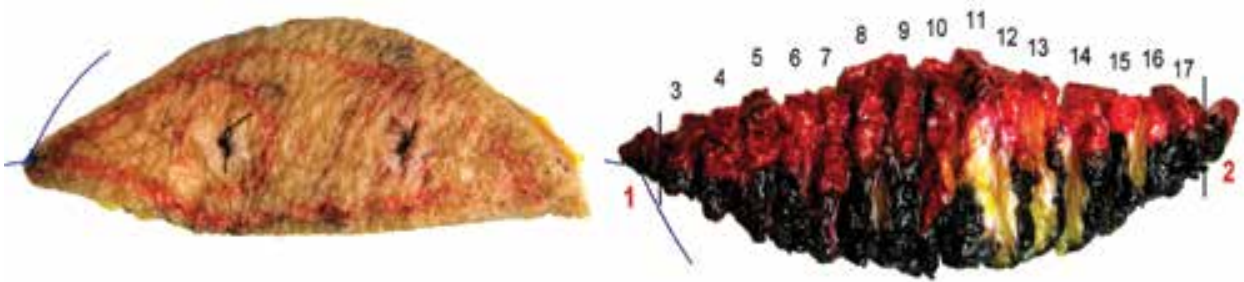
los cortes y, en el inferior, un espécimen rotulado con una seda doble en el vértice. Se hace énfasis en los cortes de los vértices numerados por aparte como 1 y 2 para control de márgenes de resección a dicho nivel y en teñir cada mitad con diferentes colores.

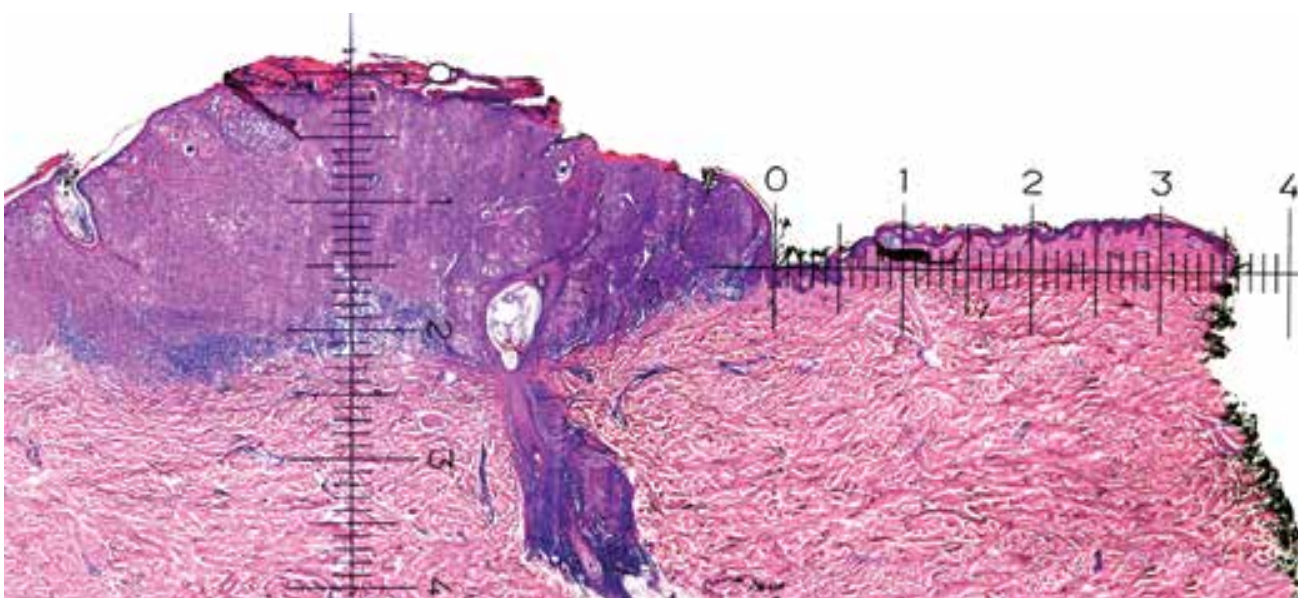

FIGURA 9. Cortes verticales de un melanoma ulcerado. Con esta técnica es fácil medir la distancia más cercana al margen lateral marcado con tinta negra (0,36 mm), al igual que la mayor profundidad de infiltración del tumor en la dermis (Breslow: 2 $\mathrm{mm})$. Hay extensión perianexial en el centro del tumor que no compromete el margen profundo, no visible en esta microfotografía. Hematoxilina y eosina, $20 \mathrm{X}$.

seborreicas y las queratosis actínicas, entre otros ejemplos $^{1,14}$. Las saucerizaciones no son más que biopsias por afeitado más profundas ${ }^{2,1}$. En ellas se produce un disco de tejido más grueso en su parte central, el cual incluye epidermis y dermis, generalmente al menos hasta su porción media o incluso hasta la grasa subcutánea, dependiendo del grosor de la piel según el área anatómica (FIGURA 7).

Comparada con la biopsia en elipse, la saucerización deja una cicatriz pequeña, redonda y más aceptable cosméticamente ${ }^{14}$.

Las muestras producto de afeitado son de difícil manejo y requieren un protocolo más elaborado. Se deben manipular suavemente para reducir el artefacto por aplastamiento y colocarse sobre papel filtro humedecido, antes de ser inmersas en formol, para evitar que se sequen, tuerzan o enrollen sobre sí mismas ${ }^{15}$. Al ser muestras muy delgadas, el agitarlas puede fragmentarlas durante la fijación y el transporte. Todos estos artefactos pueden dificultar la correcta orientación y corte del espécimen, dando origen a la posibilidad de errores en el diagnóstico histopatológico'. Tanto las biopsias por afeitado como las saucerizaciones, generalmente se dividen con cortes verticales seriados en secciones como tajadas de pan $^{12}$. Como sucede con las biopsias con sacabocados, la certeza en cuanto a la presencia o ausencia de compromiso tumoral en los márgenes de resección es baja, debido a lo delgado de la muestra que no permite evaluar márgenes profundos en paralelo (véase más adelante) y a la dificultad para su adecuado procesamiento ${ }^{2,11}$.

En el estudio de ciertos tipos de lesiones, particularmente en las lentiginosas malignas, este tipo de especímenes pueden dar origen a información incompleta al omitir la representación de focos de invasión ${ }^{2,4,16}$. Se ha encontrado que hasta el $16 \%$ de los tumores resecados, diagnosticados en la biopsia inicial como lentigos malignos, son en realidad lentigo maligno melanoma debido a la presencia de un componente invasor ${ }^{17}$. También, se ha observado un aumento importante en las tasas de recurrencia de nevus melanocíticos convencionales y displásicos, con este tipo de biopsias ${ }^{18}$. 

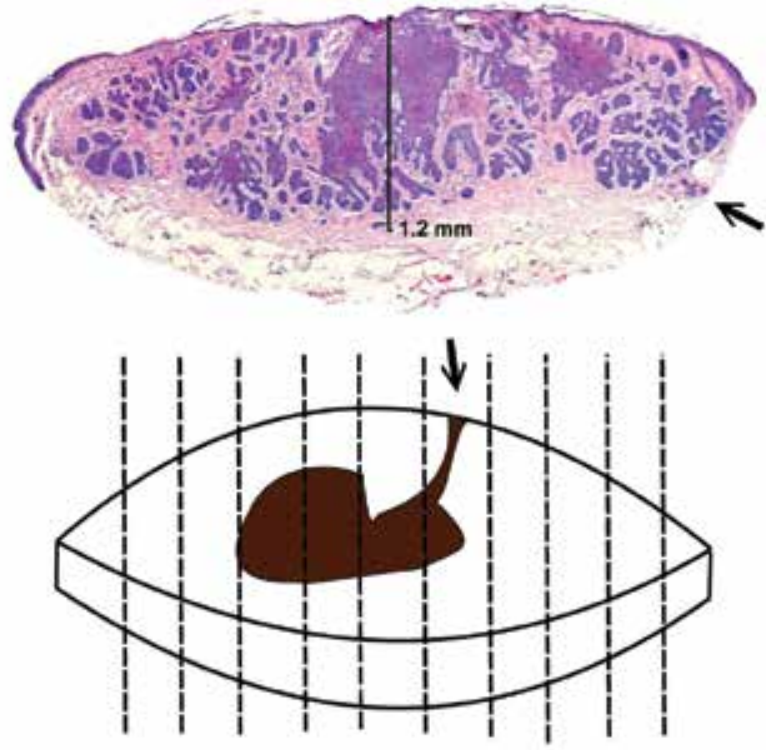

FIGURA 10. Corte en tajada de pan. Compromiso tumoral de uno de los márgenes laterales de resección en la figura superior y en uno de los puntos de los márgenes en el esquema de la figura inferior. Si no se efectúan muchos cortes seriados cuando se utiliza la técnica en tajada de pan, puede pasar desapercibida la pequeña zona de infiltración tumoral señalada con la flecha negra. Hematoxilina y eosina, 40X.

\section{Biopsias en elipse y otras similares con intención de resección completa}

Uno de los procedimientos más utilizados para practicar resecciones completas de lesiones neoplásicas es la escisión en elipse, debido a que de esta forma se dejan heridas paralelas a las líneas de tensión de la piel, lo que permite su cierre fácil y buenos resultados cosméticos. Además de las elipses alargadas, los especímenes de resección pueden presentar formas menos convencionales, como redondas, triangulares o incluso romboidales $^{10}$. Con ellas se puede efectuar el estudio de los márgenes de resección y, como veremos, se han utilizado diferentes técnicas de corte para tal fin. En un caso modelo ideal, el espécimen se recibe con los márgenes de resección con marcas de sutura o de colores para indicar su orientación espacial; luego, estos márgenes se marcan en el laboratorio con diferentes tintas, para lo cual se puede utilizar un código de colores previamente protocolizado ${ }^{8,12,19}$.

Al hacer la descripción macroscópica de la muestra se debe medir el espécimen en todas sus dimensiones y evaluar cuidadosamente la superficie cutánea, con el fin de observar la presencia lesiones asociadas, describiendo su tamaño y su relación con los márgenes de resección de la pieza estudiada. A continuación se corta la muestra. Existen varias técnicas, algunas de ellas con ventajas sobre las otras. Sin embargo, no existe la técnica perfecta que permita un $100 \%$ de seguridad al evaluar los márgenes. La certeza de que no hay tumor en los márgenes de resección depende en gran medida de un adecuado muestreo ${ }^{20,21,22}$.

Para evaluar los márgenes de resección, las técnicas más usadas en la actualidad son los cortes perpendiculares al margen quirúrgico o seriados verticales (en tajada de pan), los cortes por congelación (Mohs) y los cortes paralelos al margen quirúrgico (en face).

\section{Cortes perpendiculares al margen quirúrgico (en tajada de pan)}

Consisten en cortes verticales seriados a lo largo de todo el espécimen. Se acostumbra que las puntas o extremos de la elipse se coloquen en casetes separados (FIgURA 8) ${ }^{12}$.

Con este tipo de técnica se facilita la observación de la transición entre el tumor y la piel adyacente, por lo que es muy útil en la evaluación de lesiones melanocíticas en piel dañada por el sol que, generalmente, hacen una transición gradual y no abrupta ${ }^{20,23}$. Además, permite examinar las características morfológicas de una determinada lesión, y medir la distancia desde esta hasta el margen quirúrgico más cercano (FIGURA 9) ${ }^{24}$.

Sin embargo, esta técnica tiene la desventaja de que no permite evaluar el margen de manera completa, como se ha hecho evidente en diferentes estudios, demostrándose que al hacer los cortes verticales seriados a intervalos de 1, 2, 4 y $10 \mathrm{~mm}$, se tendría $58 \%, 37 \%$, 19 $\%$ y $7 \%$ de oportunidad de detectar márgenes positivos, respectivamente ${ }^{20,23,25}$. Para detectar cerca del $100 \%$ de los márgenes positivos, los cortes seriados tendrían que hacerse cada $0,1 \mathrm{~mm}$, lo que los hace impracticables ${ }^{25}$. Se ha encontrado que con esta técnica se pasa por alto la presencia de tumor en los márgenes quirúrgicos en porcentajes que oscilan entre el $44 \%$ y $56 \%$ de los $\operatorname{casos}^{26,27}$. Esto se debe a que los cortes seriados estándar se hacen, aproximadamente, cada 3 a $5 \mathrm{~mm}$ y cada corte sobre la lámina tiene un grosor en promedio de apenas cuatro $\mu \mathrm{m}$. En otras palabras, para detectar todas las posibles extensiones tumorales en un espécimen de $1 \mathrm{~cm}$ de longitud y haciendo cortes microscópicos cada $4 \mu \mathrm{m}$, se requerirían, aproximadamente, 2.500 cortes seriados a lo largo del bloque para examinar totalmente los márgenes (FIGURA 10) $25,27,28$. 

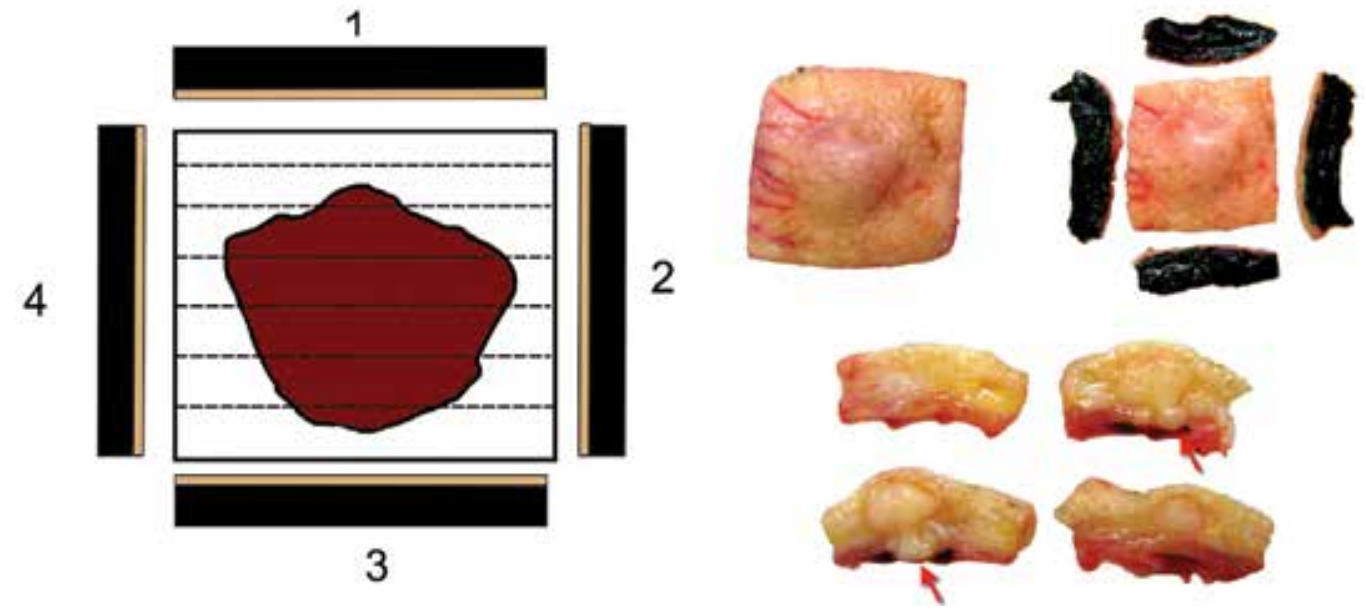

FIGURA 11. Cortes seriados en paralelo (en face sections) al margen quirúrgico de un espécimen cuadrangular, marcando con tinta negra los márgenes de resección laterales. En el lado derecho, se observa que en los cortes en tajada de pan de la porción central, el tumor se acerca al margen profundo (flecha roja).

Se concluye, entonces, que los cortes seriados transversales (en tajada de pan) pueden producir falsos negativos en el estudio de los márgenes.

\section{Cortes por congelación o cirugía micrográfica estándar de Mohs}

Debido a la complejidad de esta técnica y a que se efectúa rutinariamente por congelación, se mencionan únicamente sus aspectos más relevantes.

Frederick E. Mohs describió en 1938, mientras era un estudiante de medicina, el examen histológico completo de los márgenes de resección de muestras quirúrgicas -en lo que se conoce universalmente como cirugía micrográfica de Mohs-, llevando el conocimiento de la mejor manera de analizar un margen de resección a su punto más alto.

Desde ese momento, se han ideado varias modificaciones a esta técnica para manipular este tipo de especímenes y obtener mejores resultados en su evaluación ${ }^{22}$. El corte seriado de tejidos con el método de Mohs permite observar cerca del $100 \%$ del margen quirúrgico, incluido el margen profundo ${ }^{4,23}$. Sin embargo, con estos cortes es más difícil evaluar el detalle morfológico y la presencia de lesiones sutiles ${ }^{20,23}$.

En este tipo de cortes hay problemas técnicos importantes asociados con la congelación lenta de los tejidos y la formación de microcristales que dañan las estructuras celulares, haciendo particularmente difícil la evaluación de las secciones microscópicas de los márgenes quirúrgicos en lesiones pigmentadas de la cabeza y el cuello, debido a la presencia de melanocitos atípicos dispersos secundarios al daño actínico, que pueden ser muy complicados de diferenciar de los melanocitos neoplásicos por el artefacto mencionado ${ }^{20}$. Otro caso complejo se presenta cuando se ha desarrollado una cicatriz en el sitio de una biopsia previa, pues hace difícil diferenciar la fibrosis reactiva de un dermatofibrosarcoma en las secciones congeladas ${ }^{29}$. A pesar de sus aparentes limitaciones, es indiscutible su utilidad en la evaluación de los márgenes de resección de muchas lesiones neoplásicas ${ }^{20,23}$.

\section{Cortes seriados paralelos al margen quirúrgico (en face sections o histología 3D)}

Breuninger, et al., introdujeron la técnica de histología tridimensional completa en parafina de los márgenes de resección con cortes verticales paralelos al margen quirúrgico de resección (en face sections), que se adapta muy bien a las condiciones de cualquier laboratorio ${ }^{22}$. Se conoce también como técnica del polígono y es una modificación de la cirugía micrográfica de Mohs, que puede realizarse por congelación o mediante fijación en formol e inclusión en parafina, para producir cortes permanentes coloreados con hematoxilina y eosina ${ }^{16,22,29}$. Esta técnica permite examinar la mayor parte del margen quirúrgico con un relativamente bajo número de cortes $^{23}$.

En ella se hacen cortes paralelos a los márgenes de 

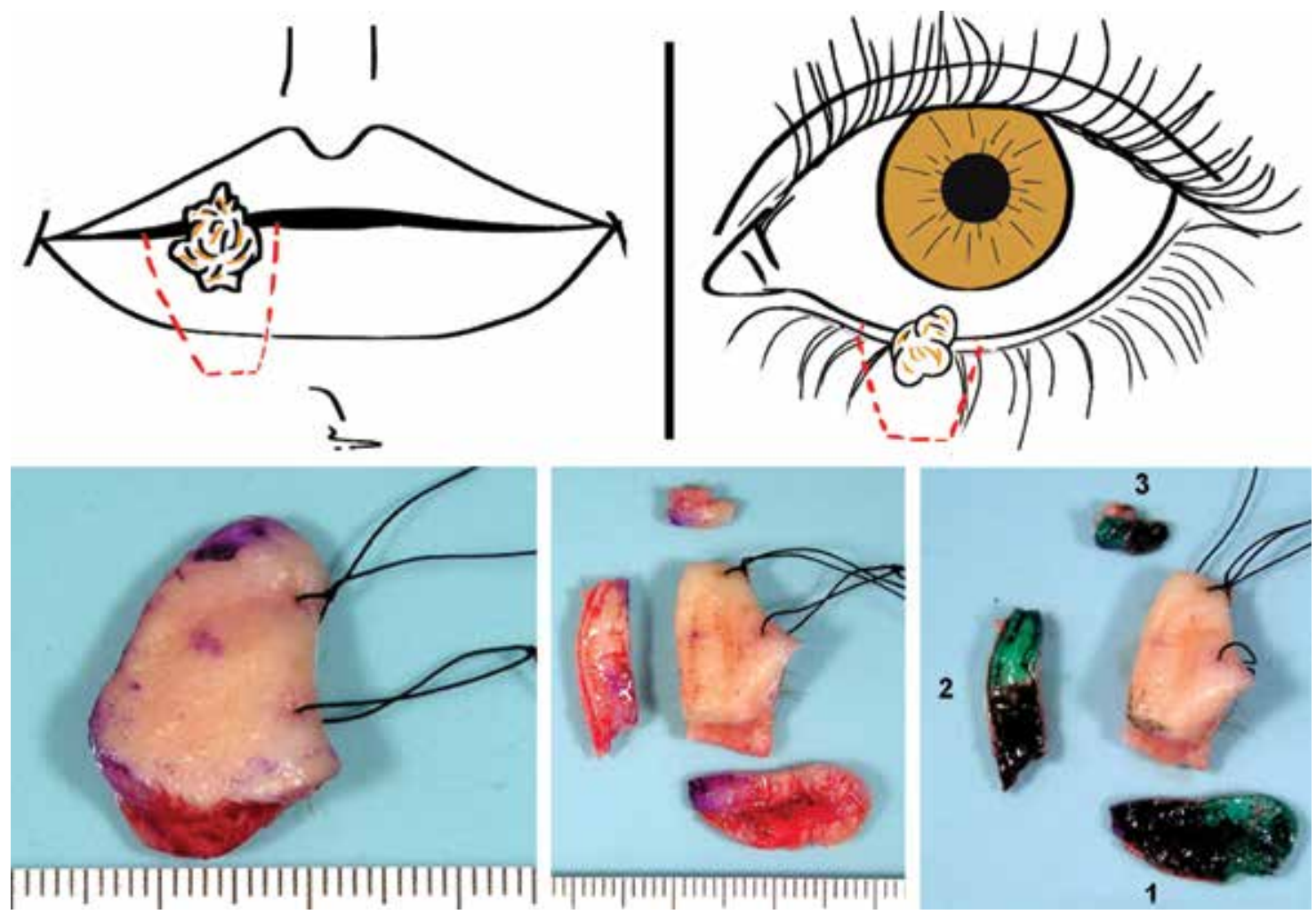

FIGURA 12. Cortes en paralelo al margen quirúrgico de resección en un borde libre. En la parte superior, esquema de lesiones tumorales extirpadas en cuña. En la parte inferior, procesamiento de una extirpación en cuña del pabellón auricular. Se hacen cortes seriados delgados de cada borde y cada mitad se tiñe con diferentes colores.

resección, marcando con tinta la periferia de los márgenes laterales y el profundo, con el fin de que el técnico haga una inclusión adecuada y realmente se observe el margen quirúrgico en las placas resultantes. Los fragmentos se identifican individualmente o se introducen separadamente en casetes histológicos y son preparados para cortes por congelación o en parafina ${ }^{30}$. Esta técnica puede emplearse en especímenes de diferente forma y tamaño, aplicando los mismos principios. La porción sobrante central de la muestra, carente de márgenes de resección laterales y profundo, se secciona en cortes verticales seriados en tajada de pan a intervalos de $3 \mathrm{~mm}$, con el fin de apreciar el compromiso tumoral de la epidermis, la dermis y el tejido celular subcutáneo ${ }^{17 .}$ Si se detectan nidos de células neoplásicas en los márgenes, se toman pasos adicionales de ampliación quirúrgica de dichos márgenes y se procesan de igual manera (FIGURA 11) ${ }^{30}$.

Para este procedimiento se requiere un cambio en el pensamiento con respecto a las ideas tradicionales, pasando del concepto del tamaño del margen que debe ser resecado, al concepto de márgenes verdaderamente libres histológicamente. Es por esto que se recomienda el completo control histológico de los márgenes quirúrgicos enteros, usando la orientación tisular paralela al margen (en face), con el fin de reducir el riesgo de recurrencia ${ }^{25}$. De esta forma, se puede alcanzar el control tumoral local con una reducción simultánea de los márgenes de resección en, aproximadamente, dos terceras partes, sin incremento en el riesgo de recurrencias locales y con mejor pronóstico; además, permite alcanzar excelentes resultados cosméticos y funcionales ${ }^{22,30}$.

El procesamiento en paralelo al margen quirúrgico (en face) tiene como desventaja el hecho de no permitir la medición de la distancia entre el tumor y el margen quirúrgico.

Los casos de lentigo maligno y lentigo maligno melanoma manejados con esta técnica, son más fáciles de evaluar usando secciones fijadas en formol e incluidas en parafina de la manera usual. Esta técnica también se ha usado en el manejo de dermatofibrosarcoma protuberans, angiosarcoma y fibroxantoma atípico ${ }^{29}$. Este tipo de corte también se puede practicar muy fácilmente en los especímenes de bordes semimucosos o del borde libre de la oreja (FIGURA 12). 

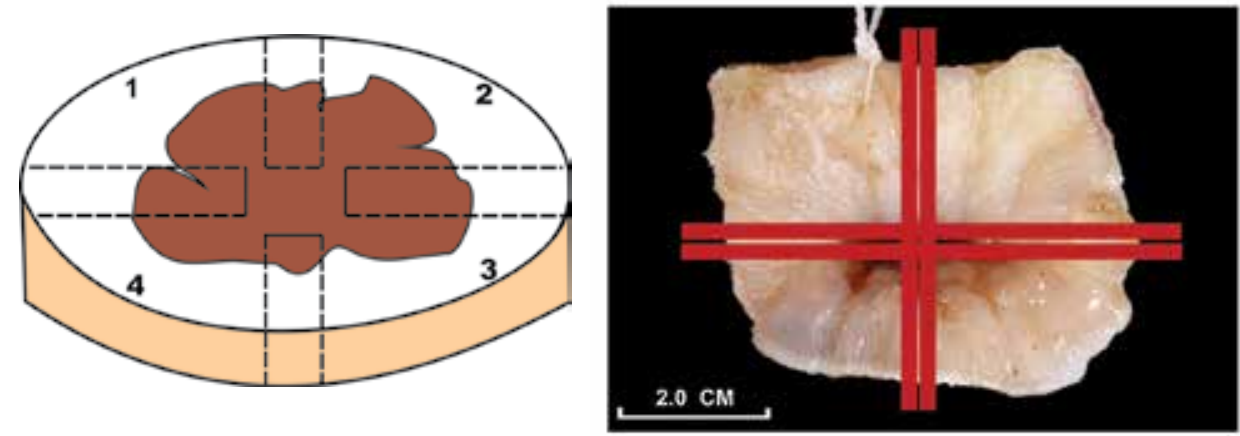

FigURA 13. Cortes en cruz. A la izquierda, piel. Con esta técnica se dejan de evaluar las zonas de las esquinas $(1,2,3,4)$ laterales y profundas.

A la derecha, escisión en cono de cuello uterino por un carcinoma escamocelular in situ.

A ningún patólogo se le ocurriría procesar, por igual razón, un cuello uterino con solo cuatro cortes en cruz.

\section{Cortes en cruz}

Infortunadamente, todavía se utiliza en muchos laboratorios de patología general la técnica de cortes radiales en cruz para evaluar los márgenes de resección en especímenes de piel que albergan lesiones tumorales malignas. De todos los estilos descritos anteriormente de cómo procesar una biopsia de piel, este es el menos recomendable, ya que se dejan de evaluar extensas áreas del espécimen con potencial compromiso tumoral (FIGURA 13).

\section{Biopsias de cuero cabelludo}

El procesamiento de este tipo de biopsias ya fue revisado en extenso en otro artículo de esta revista. Aquí se mencionan los aspectos más relevantes y recientes al respecto ${ }^{31}$. El corte en secciones de especímenes de cuero cabelludo orientado al diagnóstico de alopecias, tuvo un cambio fundamental en 1984, cuando Headington publicó los resultados de su estudio "Anatomía microscópica transversa del cuero cabelludo humano", en el que sugirió el corte en secciones transversales como un método nuevo y ventajoso para la evaluación y el diagnóstico de este tipo de especímenes ${ }^{32,33}$.

En la actualidad, se recomienda tomar dos biopsias con sacabocados de $4 \mathrm{~mm}$. Es responsabilidad del dermatólogo tomar la muestra adecuadamente, o sea, paralela a la dirección de crecimiento del pelo, y no menos importante, escoger la zona más activa, con el fin de maximizar los hallazgos que permitan hacer un diagnóstico certero ${ }^{33,34}$.

\section{Cortes horizontales}

Son los tipos de corte más recomendados cuando se sospecha una alopecia difusa. En este tipo de alopecia ambas biopsias deben ser cortadas en sentido transversal (horizontal), aproximadamente, $2 \mathrm{~mm}$ por debajo de la epidermis, para que el histotecnólogo haga cortes sucesivos hacia la epidermis y el tejido celular subcutáneo. Algunos autores proponen hacer tres o cuatro secciones transversas en la muestra, con el fin de permitir el examen de múltiples niveles en una lámina ${ }^{34}$.

Las secciones horizontales proveen un material histológico de gran calidad para análisis morfométricos y cuantitativos, incluyendo la cuantificación de folículos terminales o miniaturizados, al igual que las distintas fases del ciclo normal de crecimiento del pelo. La mayor ventaja es que se aprecian muchos folículos pilosos en el mismo plano de corte, que pueden ser contados y examinados en diferentes niveles y en pocas placas histológicas (FIGURA 14). Son especialmente útiles en la evaluación de las formas no cicatriciales de alopecia, particularmente en alopecias de tipo andrógeno masculino y femenino, efluvio telógeno, tricotilomanía y algunas formas poco frecuentes de alopecia areata difusa ${ }^{35}$.

$\mathrm{Su}$ principal limitación es la poca representación de la unión dermoepidérmica y la epidermis como tal $^{31,33,34,35}$. En realidad, puede ser difícil o totalmente imposible diagnosticar una psoriasis, un liquen simple crónico, una foliculitis decalvans o una alopecia lipedematosa, si no se dispone de una biopsia seccionada verticalmente.

\section{Cortes verticales}

Las secciones verticales tienen la ventaja de que son más fáciles de hacer para el técnico y de interpretar para el patólogo, ya que corresponden a los cortes de rutina utilizados cotidianamente en los laboratorios de patología. Son una excelente herramienta que permite diagnosticar muchas de las alopecias y no debería descartarse. Con esta técnica se obtiene una óptima visualización de la epidermis, la unión dermoepidérmica 

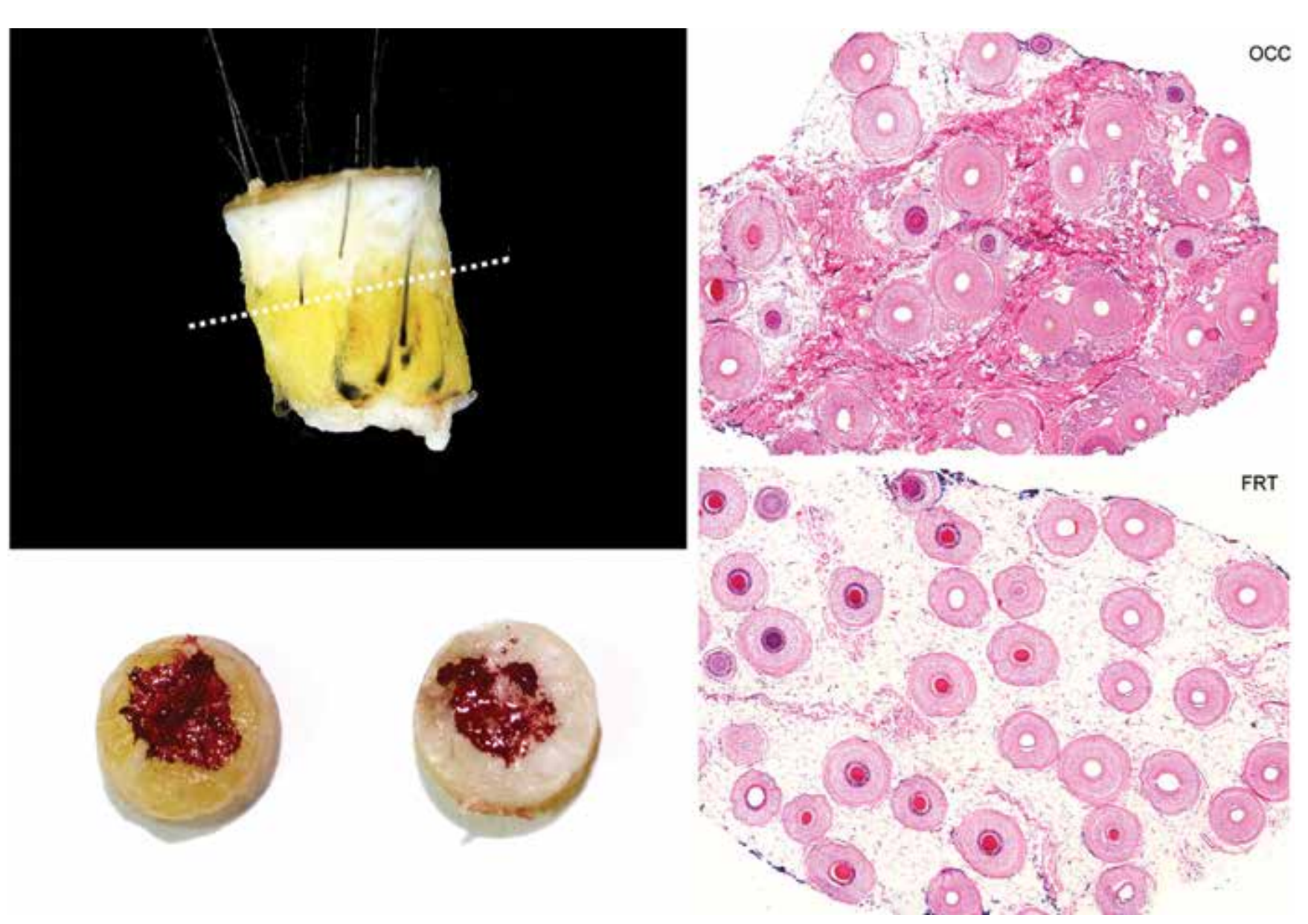

FIGURA 14. Biopsia con sacabocados de cuero cabelludo. En la parte superior izquierda, se aprecia un espécimen tomado con sacabocados, para corte horizontal. En la parte inferior izquierda, se aprecian los fragmentos obtenidos. La marca con tinta roja se toma como guía para que el histotecnólogo comience el corte en ese lado. A la derecha, se aprecian los cortes transversales comparativos, de la zona occipital y la frontal.

y la dermis superior, muy útil para el estudio de las enfermedades que afectan la interface y el segmento superior del folículo piloso, como el lupus eritematoso discoide, la psoriasis y, en general, las alopecias cicatriciales ${ }^{33,36}$. Una desventaja de esta técnica radica en los pocos folículos que se observan en un solo corte, y lo segmentario y oblicuo de los tallos pilosos seccionados, por lo que se requieren numerosos cortes para visualizar un folículo completo en todos sus segmentos. No es una forma de corte adecuada para hacer estudios cuantitativos y relaciones numéricas entre las distintas fases en la que se encuentren los folículos pilosos (anágeno-telógeno, terminales-vellos, etc.); por lo tanto, su utilidad se ve muy limitada en el diagnóstico de las alopecias de tipo andrógeno y el efluvio telógeno crónico ${ }^{31,34}$.

La tendencia actual para alcanzar el máximo rendi- miento diagnóstico es utilizar cortes verticales y horizontales, siempre y cuando el dermatólogo tome dos biopsias y el diagnóstico clínico no sea una alopecia andrógena, una alopecia areata difusa o un efluvio telógeno, en los cuales se debe utilizar el corte transversal para ambas biopsias ${ }^{31,33,34}$.

Una técnica adicional para procesamiento de muestras de cuero cabelludo es la técnica HoVert, la cual permite hacer cortes verticales y horizontales de un único fragmento de biopsia. Con esta técnica, una biopsia con sacabocado de $4 \mathrm{~mm}$ se corta horizontalmente a un $\mathrm{mm}$ por debajo de su superficie, creándose así un disco epidérmico y una porción inferior. El disco epidérmico se somete a cortes verticales seriados y se incluye, para obtener secciones verticales estándar. La porción inferior se somete a cortes horizontales seriados para obtener secciones transversales ${ }^{37}$. 

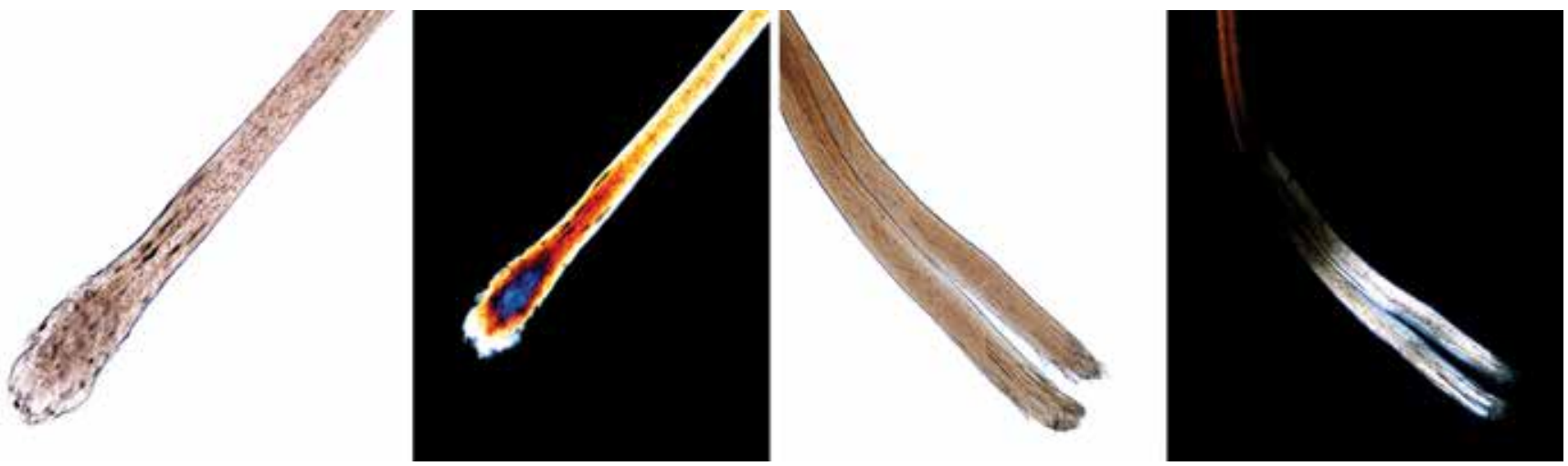

FIGURA 15. A la izquierda, tallo y bulbo piloso en telógeno. A la derecha, tallo piloso distal con tricoptilosis. Ambos especímenes han sido colocados en una lámina y mantenidos en su lugar con el medio de montaje usual y una laminilla cubreobjetos. Esta técnica permite una gran resolución con microscopía de luz y luz polarizada (imágenes en fondo oscuro).

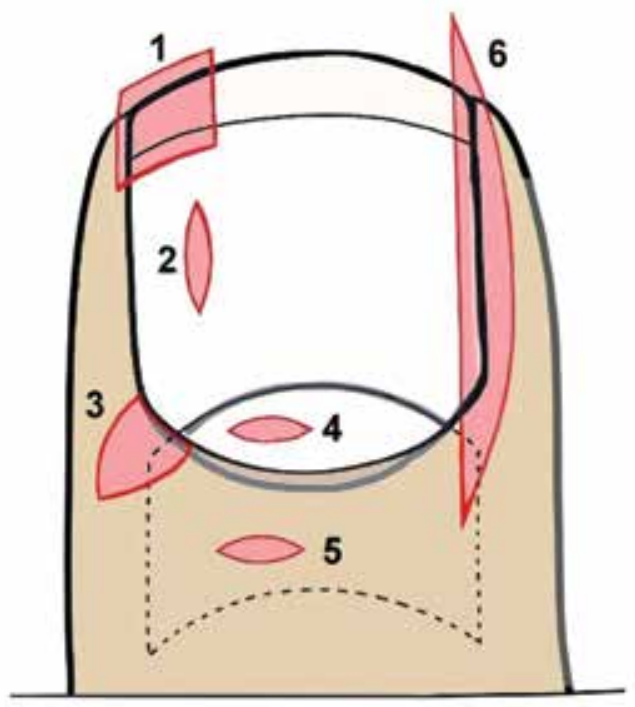

FIGURA 16. Biopsias de uña. Se ilustra la localización de los diferentes sitios en los que se puede practicar la biopsia: 1) lámina, 2) lecho, 3) pliegue; 4) matriz distal, 5) matriz proximal y 6) longitudinal.

\section{Tallos pilosos}

En general, puede decirse que en dermatopatología este tipo de muestras se toman con dos propósitos: el estudio de las enfermedades del tallo piloso y el estudio de la fase del ciclo.

\section{Estudio de las enfermedades del tallo piloso}

En estos casos las muestran deben tomarse de áreas afectadas y no afectadas, cortando suavemente el ex- tremo más proximal del tallo, a ras del cuero cabelludo, con una tijera curva. Es muy importante evitar el más mínimo trauma por arrancamiento o aplastamiento. La muestra obtenida se puede colocar sobre un portaobjetos, cubriéndola con cinta adhesiva ${ }^{38,39}$. También, se pueden cubrir con el medio de montaje de rutina utilizado en el laboratorio y, encima, con una laminilla o cubreobjetos. No se debe ejercer presión, con el fin de evitar artefactos $4^{\circ}$. Los cambios más específicos están en la porción proximal. Siempre se debe utilizar luz polarizada, particularmente en los casos de niños con alopecia ocasionada por pelo corto y frágil en quienes se sospeche una tricotiodistrofia.

\section{Estudio de la fase del ciclo}

Para estudiar las fases del ciclo se debe observar el bulbo piloso. La muestra se puede obtener de los cabellos que haya perdido el paciente o, más frecuentemente, mediante tracción suave o forzada (tricograma). En los casos de anagen suelto, puede ser útil teñir los tallos pilosos con azul de toluidina o elástico de van Gieson, para visualizar mejor la vaina radicular interna ${ }^{41,42,43,44}$. En estos casos, el montaje de los tallos pilosos con los bulbos en su extremo proximal es similar al descrito anteriormente para el estudio de las enfermedades del tallo piloso (FIGURA 15).

No hay que olvidar tampoco montar algunos tallos en hidróxido de potasio al $20 \%$ y cultivar en agar de Sabourau para descartar una infección por hongos.

\section{Biopsias de uña}

Solo unos pocos estudios con un número limitado de pacientes se han llevado a cabo para verificar la utilidad de la biopsia de uña $45,46,47,48$. 


\begin{tabular}{lll}
\multicolumn{1}{c}{ Tipo de biopsia } & \multicolumn{1}{c}{ Indicaciones } & \multicolumn{1}{c}{ Procedimiento } \\
Lámina ungular & $\begin{array}{l}\text { Onicomicosis, pigmentos, psoriasis, } \\
\text { distrofias ungulares } \\
\text { Onicomicosis, psoriasis, melanoma, } \\
\text { enfermedad de Bowen, glomangioma, } \\
\text { osteocondroma }\end{array}$ & $\begin{array}{l}\text { Biopsia distal, laminectomía y con } \\
\text { sacabocado }\end{array}$ \\
Lecho ungular & $\begin{array}{l}\text { Psoriasis, liquen plano, lesiones } \\
\text { pigmentadas y otras lesiones neoplásicas }\end{array}$ & $\begin{array}{l}\text { Biopsia con sacabocados y biopsia en elipse } \\
\text { o longitudinal }\end{array}$ \\
Mnatriz ungular & $\begin{array}{l}\text { Melanoniquia longitudinal, psoriasis, liquen } \\
\text { plano, lesiones pigmentadas y otras lesiones } \\
\text { neoplásicas, uña encarnada }\end{array}$ & Biopsia longitudinal lateral
\end{tabular}

TABLA 1. Indicaciones y tipo de biopsia ungular según el área afectada.

Si bien aún existe algo de resistencia a tomar este tipo de biopsia, cada vez hay más autores que la recomiendan ${ }^{49,50,51}$. Realmente, no es muy difícil de hacer. No obstante, requiere un conocimiento profundo de la anatomía y fisiología de la unidad ungular, al igual que experiencia quirúrgica, y un patólogo entrenado y motivado para su interpretación ${ }^{52}$. Más que en cualquier otro tipo de biopsia, el éxito o fracaso del procedimiento descansa en una selección muy adecuada del tipo de biopsia empleada, la selección del sitio más representativo de la enfermedad y la remisión adecuada de la muestra al laboratorio de patología. Dada su importancia, se discute en extenso esta fase preanalítica del estudio de la biopsia de uña (TABLA 1) 49,53,54,55,56.

Las modalidades de biopsia más empleadas son la de lámina ungular, la de lecho ungular, la de matriz ungular, la de pliegue ungular y la longitudinal de piel, matriz y lecho ungular (FIGURA 16).

Biopsia de la lámina ungular. Puede hacerse mediante corte de la porción distal de la lámina con unas tijeras o un cortaúñas y recolección de los detritos por debajo de la misma en el hiponiquio. Es un material muy útil para el diagnóstico de onicomicosis. La otra técnica es obtener la lámina ungular completa ${ }^{57,58}$.

\section{Biopsia del lecho ungular}

Después de la extirpación de la lámina ungular, se puede obtener una biopsia fusiforme por escisión. Esta biopsia también se puede obtener a través de la lámina ungular, usando la técnica del doble sacabocados. La morbilidad y la cicatrización resultantes son mínimas ${ }^{59}$.

\section{Biopsia de la matriz ungular}

Cuando se necesita evaluar una melanoniquia, lo mejor es obtener una biopsia de la matriz ungular. La técnica es un poco más compleja que las anteriormente enunciadas $\mathrm{y}$, al igual que la del lecho, requiere retirar la lámina ungular y exponer la matriz. Puede conducir a daño grave con consecuencias estéticas desagradables, al poder producir deformación de la lámina ungular. Para minimizar esto, es mejor tomar la biopsia de la matriz distal, ya que allí hay un menor riesgo de originar distrofia. Además, la matriz distal es el sitio donde hay mayor concentración de melanocitos y, por consiguiente, la mayoría de las lesiones pigmentadas se originan allí6o.

\section{Biopsia del pliegue ungular}

Puede tomarse del pliegue proximal o del pliegue lateral y está indicada en dermatosis con paroniquia o tumores benignos o malignos.

\section{Biopsia longitudinal de piel, matriz y lecho ungular}

Es la muestra ideal para estudio histopatológico, particularmente cuando hay una lesión melanocítica, ya que incluye todas las estructuras del aparato ungular: matriz, pliegue proximal, lecho e hiponiquio. El tejido se diseca en bloque y se remite al patólogo con las marcas de las zonas representadas ${ }^{61}$. 

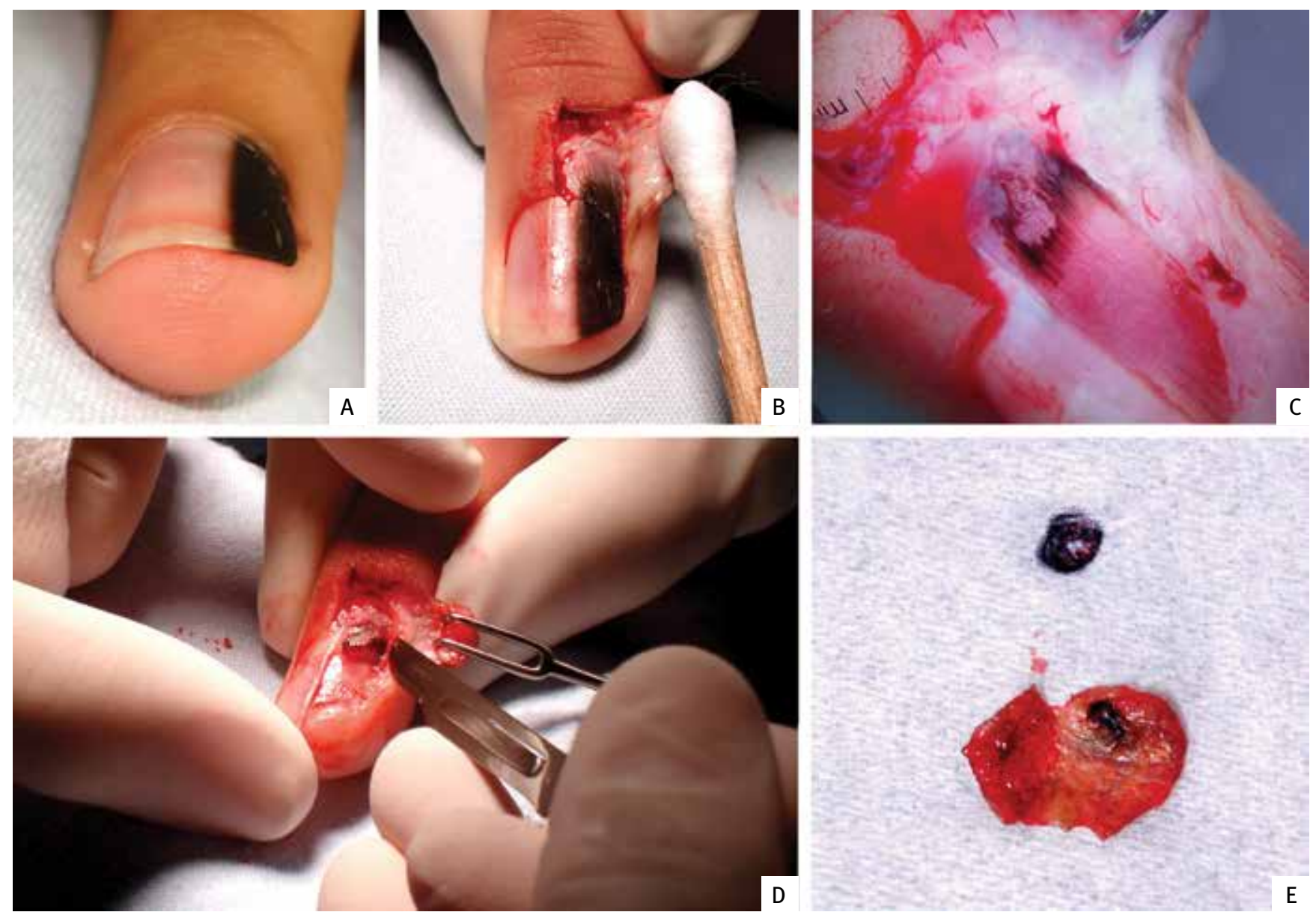

FIGURA 17. Biopsia longitudinal. A. Melanoniquia estriada. B. Incisión en forma de "L". Se separa el tejido sobre el área pigmentada y se identifica la lesión que origina el pigmento. C. La dermatoscopia muestra una lesión pigmentada sobre la matriz proximal y la lúnula. D. Se diseca el área comprometida por el pigmento, tomando todo el lecho. E. El material obtenido debe colocarse sobre papel, con una marca puntual con tinta seca (arriba) que muestre la localización de la matriz ungular.

\section{Procesamiento}

El conocimiento de la histopatología de las enfermedades de las uñas ha sido mucho más limitado que el de otras enfermedades cutáneas, no solamente por las pocas biopsias que se practican, sino porque la uña tiene una estructura mucho más compleja y una forma más limitada de reaccionar a las enfermedades que el resto de los tejidos cutáneos.

Las muestras deben ser meticulosamente orientadas en la sala de cirugía y el dermatopatólogo debe mantener esta orientación a lo largo de todo el proceso. El problema más común e importante que puede presentarse al procesar una biopsia de uña, es perder la correcta orientación en el material incluido. Esto se puede evitar marcando en forma adecuada el espécimen para guiar su posición en el bloque de parafina.
El dermatólogo debe suministrarle al patólogo toda la información clínica sobre la sospecha de la enfermedad que puede padecer el paciente, al igual que una descripción precisa de la técnica de biopsia y el sitio exacto de la toma. Debe ayudarse de un diagrama que oriente espacialmente el espécimen, ojalá usando un fragmento de papel de filtro para adherir la biopsia allí mismo y poder dibujar las marcas requeridas (FIGURA 17).

Debido a lo delgado del epitelio, a la presencia de una dermis formada prácticamente por tejido tendinoso que se inserta en la falange distal y a la ausencia de tejido celular subcutáneo en la unidad ungular, cualquier biopsia de este sitio no tendrá como punto de referencia la grasa subcutánea, sino que debe llegar hasta el periostio. Esto hace que el procedimiento sea difícil tanto para el dermatólogo como para el patólogo que la debe manejar. Por esta misma razón, las biopsias no se ad- 
hieren adecuadamente al papel de filtro y, si no se fijan con un hilo o se encierran adecuadamente en el papel, llegan sueltas en el frasco al laboratorio, y se pierde su orientación.

Se debe tener presente que el material formado por queratina de la lámina ungular debe ablandarse previamente. Hay muchas técnicas y soluciones ablandadoras. Una buena opción es hacerlo, previa fijación en formol tamponado neutro al $10 \%$, en solución de $\mathrm{KOH}$ al $20 \%$, Tween 40 al $10 \%$, o en ácido nítrico diluido al $10 \%$, por una o dos horas, según el tamaño y la dureza de la muestra, teniendo cuidado de que no se ablande demasiado y finalmente se pulverice. A continuación se puede lavar con agua para retirar la solución ablandadora y guardar de nuevo en formol tamponado neutro al $10 \%$, para su posterior inclusión en parafina y sección con cortes transversales ${ }^{62,63,64}$. Finalmente, todas las muestras de tejidos blandos o fragmentos de uña ya ablandados en $\mathrm{KOH}$, se tiñen inicialmente con hematoxilina y eosina, ácido peryódico de Schiff (PAS), con diastasa o sin ella, y opcionalmente con plata metenamina. Además, se puede utilizar inmunohistoquímica con $\mathrm{S} 100$ y HMB45, cuando se piensa en una lesión pigmentada. Muchos otros marcadores también pueden ser útiles si se está frente a una lesión de tejidos blandos acrales.

Es importante tener en cuenta que hay particularidades muy especiales en la histopatología de la uña, tanto la normal como aquella con alteraciones patológicas propiamente dichas.

Algunas de las características más significativas son las que se mencionan a continuación.

La matriz ungular no tiene capa granulosa y produce la lámina ungular mediante queratinización onicolemal.

No se puede olvidar que hay una mayor densidad de melanocitos en la matriz distal que en la proximal, ya que esta se encuentra protegida del sol por el pliegue proximal.

De igual trascendencia es que los melanocitos no son exclusivamente basales, sino que pueden visualizarse normalmente hasta la tercera capa de células epiteliales, en una disposición pseudopagetoide, particularmente en los melanocitos de localización proximal.

Otra característica muy particular es que los melanocitos intraepiteliales son negativos para el marcador $\mathrm{S} 100^{65,66}$.

La matriz proximal da lugar a la cara dorsal de la uña y la matriz distal da lugar a la cara ventral de la lámina ungular, por lo que un examen dermatoscópico del borde libre de la lámina puede brindar información valiosa sobre la localización del pigmento en la uña y, por ende, en la matriz.

El epitelio del lecho ungular puede ser de solamente dos o tres capas de espesor. No tiene capa granulosa y carece de red de crestas cuando el corte se efectúa en sentido longitudinal.

Un factor que debe tenerse en cuenta es que, al estar el lecho ungular formado por un epitelio tan delgado, cuando se extrae la lámina ungular existe el riesgo de que pueda quedar adherido del lado ventral de la lámina ungular y no en la biopsia del lecho propiamente dicha. Por esta razón, siempre es prudente no desechar la lámina ungular y examinarla como cualquier otro tejido ${ }^{67}$.

\section{Conclusión}

El trabajo del dermatopatólogo es un trabajo en equipo con el dermatólogo y depende de manera directa de la información suministrada por el médico tratante ${ }^{15}$. Sin estos datos, el patólogo navegará a la deriva en el estudio de un caso para el cual dictaminará uno o varios diagnósticos que pueden o no relacionarse clínicamente. No es mala idea que el dermatopatólogo observe clínicamente al paciente y se forme una idea de sus problemas.

Además, son varias las expectativas que el dermatólogo tiene del estudio de los especímenes cutáneos remitidos. El dermatopatólogo debe hacer un informe claro y preciso. La práctica de reportar márgenes está influenciada predominantemente por el diagnóstico histológico, siendo por ejemplo este reporte más común en melanomas que en nevus ${ }^{68}$. Sin embargo, los dermatopatólogos deben utilizar prácticas estandarizadas y un lenguaje común a la hora de reportar los márgenes quirúrgicos y los dermatólogos clínicos deben reconocer las limitaciones de la evaluación de dichos márgenes según el tipo de espécimen que ellos envíen para estudio histopatológico ${ }^{11,45}$.

\section{Agradecimientos}

A la Dra. Marcela Carreño, por los diagramas de las figuras 3, 5, 8, 10, 11 y 13; a la Dra. Luz Marina Gómez, por la composición fotográfica de la figura $17 \mathrm{y}$ al Dr. Miguel Gaitán, por los diagramas utilizados en las figuras 2, 12 y 16.

\section{Referencias}

1. Sina B, Kao GF, Deng AC, Gaspari AA. Skin biopsy for inflammatory and common neoplastic skin diseases: Optimum time, best location and preferred techniques. A critical review. J Cutan Pathol. 2009;36:505-10.

2. Martin RC, Scoggins CR, Ross MI, Reintgen DS, Noyes RD, Edwards MJ, et al. Is incisional biopsy of melanoma harmful? Am J Surg. 2005;190:913-7.

3. Ng JC, Swain S, Dowling JP, Wolfe R, Simpson P, Kelly JW. The im- 
pact of partial biopsy on histopathologic diagnosis of cutaneous melanoma: Experience of an Australian tertiary referral service. Arch Dermatol. 2010;146:234-9.

4. Weisberg NK, Becker DS. Potential utility of adjunctive histopathologic evaluation of some tumors treated by Mohs micrographic surgery. Dermatol Surg. 2000;26:1052-6.

5. Hennessy J, Clarke LE, Ioffreda MD, Helm KF. Enhancement of the diagnostic accuracy of large skin excision pathology reports by adding gross specimen photographs. J Cutan Pathol. 2009;36:711-2.

6. Blasdale C, Charlton FG, Weatherhead SC, Ormond P, Lawrence CM. Effect of tissue shrinkage on histological tumour-free margin after excision of basal cell carcinoma. Br J Dermatol. 2010;162:60710.

7. Kerns MJ, Darst MA, Olsen TG, Fenster M, Hall P, Grevey S. Shrinkage of cutaneous specimens: Formalin or other factors involved? J Cutan Pathol. 2008;35:1093-6.

8. Paterson DA, Davies JD, Mclaren KM. Failure to demonstrate the true resection margins of excised skin tumours: A case for routine marking. Br J Dermatol. 1992;127:119-21.

9. Chang TT, Somach SC, Wagamon K, Somani AK, Pomeranz J, Jaworsky C, et al. The inadequacy of punch-excised melanocytic lesions: Sampling through the block for the determination of "margins". J Am Acad Dermatol. 2009;60:990-3.

10. Westra WH, Hruban RH, Phelps TH, Isacson C. Surgical pathology dissection: An illustrated guide. Second edition. New York: Springer; 2003.

11. Sellheyer K, Bergfeld WF. When to ask your dermatopathologist to evaluate for surgical margins and when not: A matter of confusion for dermatologists and dermatopathologists alike. J Am Acad Dermatol. 2005;52:1095-7.

12. Kolman O, Hoang MP, Piris A, Mihm MC, Duncan LM. Histologic processing and reporting of cutaneous pigmented lesions: Recommendations based on a survey of 94 dermatopathologists. J Am Acad Dermatol. 2010;63:661-7.

13. Bruecks AK, Shupe JM, Trotter MJ. Prospective step sections for small skin biopsies. Arch Pathol Lab Med. 2007;131:107-11.

14. Pickett $H$. Shave and punch biopsy for skin lesions. Am Fam Physician. 2011;84:995-1002.

15. Alavi A, Niakosari F, Sibbald RG. When and how to perform a biopsy on a chronic wound. Adv Skin Wound Care. 2010;23:132-40.

16. Ariza SA, Acosta A. Lentigo maligno: clínica, histopatología y tratamiento. Rev Asoc Col Dermatol. 2008;16:135-42.

17. Hazan C, Dusza SW, Delgado R, Busam KJ, Halpern AC, Nehal KS. Staged excision for lentigomaligna and lentigomaligna melanoma: A retrospective analysis of 117 cases. J Am Acad Dermatol. 2008;58:142-8.

18. Sommer LL, Barcia SM, Clarke LE, Helm KF. Persistent melanocytic nevi: A review and analysis of 205 cases. J Cutan Pathol. 2011;38:503-7.

19. Dayton AS, Ro JY, MD, Schwartz MR, Ayala AG, Raymond AK. Raymond's paragraph system: An alternative format for the organization of gross pathology reports and its implementation in an academic teaching hospital. Arch Pathol Lab Med. 2009;133:298302.

20. Prieto VG, Argeny ZB, Barnhill RL, Duray PH, Elenitsas R, From L, et al. Are en face frozen sections accurate for diagnosing margin status in melanocytic lesions? Am J Clin Pathol. 2003;120:203-8.

21. Weinstein MC, Brodell RT, Bordeaux J, Honda K. The art and sci- ence of surgical margins for the dermatopathologist. Am J Dermatopathol. 2012;34:737-45.

22. Mohs FE. Chemosurgery. A microscopically controlled method of cancer excision. Arch Surg. 1941;42:279-95.

23. Trotter MJ. Melanoma margin assessment. Surgical Pathology 2009;2:543-52.

24. Scolyer RA, Judge MJ, Evans A, Frishberg DP, Prieto VG, Thompson JF, et al. Data set for pathology reporting of cutaneous invasive melanoma: Recommendations from the international collaboration on cancer reporting (ICCR). Am J Surg Pathol. 2013;37:1797814. Fecha de consulta: 3 de enero de 2014. Disponible en: http:// www.ncbi.nlm.nih.gov/pmc/articles/PMC3864181/.

25. Kimyai-Asadi A, Katz T, Goldberg LH, Ayala GB, Wang SQ, Vujevich JJ, et al. Margin Involvement after the excision of melanoma in situ: The need for complete en face examination of the surgical margins. Dermatol Surg. 2007;33:1434-9.

26. Kimyai-Asadi A, Alam M, Goldberg LH, Peterson SR, Silapunt S, Jih MH. Efficacy of narrow-margin excision of well-demarcated primary facial basal cell carcinomas. J Am Acad Dermatol. 2005;53:464-8.

27. Kimyai-Asadi A, Goldberg LH, Jih MH. Accuracy of serial transverse cross-sections in detecting residual basal cell carcinoma at the surgical margins of an elliptical excision specimen. J Am Acad Dermatol. 2005;53:469-74.

28. Thomas DJ, King AR, Peat BG. Excision margins for nonmelanotic skin cancer. Plast Reconstr Surg. 2003;112:57-63.

29. Clayton BD, Leshin B, Hitchcock MG, Marks M, White WL. Utility of rush paraffin-embedded tangential sections in the management of cutaneous neoplasms. Dermatol Surg. 2000;26:671-8.

30. Lichte V, Breuninger H, Metzler G, Haefner HM, Moehrle M. Acral lentiginous melanoma: Conventional histology Vs. three-dimensional histology. Br J Dermatol. 2009;160:591-9.

31. Restrepo R. Cortes transversales Vs. verticales para el diagnóstico de las alopecias. Rev Asoc Col Dermatol. 2008;16:23-8. Fecha de consulta: 1 de enero de 2014. Disponible en: http://revistasocolderma.org/files/Articulo\%20de\%2orevision \%20-\%20Cortes $\% 20$ transversales\%20vs\%20verticales.pdf.

32. Flotte TJ. Transverse sectioning of the scalp (Headington technique) in the 19th century. J Cutan Pathol. 2008;35:82-5.

33. Bôer A, Hoene K. Transverse sections for diagnosis of alopecia? Am J Dermatopathol. 2005;27:348-52.

34. Elston DM, Ferringer T, Dalton S, Fillman E, Tyler W. A comparison of vertical versus transverse sections in the evaluation of alopecia biopsy specimens. J Am Acad Dermatol. 2005;53:267-72.

35. Bathish N, Izhak OB, Shemer A, Bergman R. A study of serial vertical sectioning of scalp biopsies to increase the histological diagnostic yield in alopecias. J Eur Acad Dermatol Venereol. 2010;24:709-15

36. García C, Poletti E. Scalp biopsy specimens: Transverse Vs. vertical sections. Arch Dermatol. 2007;143:268.

37. Nguyen JV, Hudacek K, Whitten JA, Rubin AI, Seykora JT. The HoVert technique: A novel method for the sectioning of alopecia biopsies. J Cutan Pathol. 2011;38:401-6.

38. Shelley WB. Hair examination using double-stick tape. J Am Acad Dermatol. 1983;8:430-1.

39. Whiting DA, Dy LC. Office diagnosis of hair shaft defects. Semin Cutan Med Surg. 2006;25:24-34.

40. Zitelli JA. Pseudomonilethrix. An artifact. Arch Dermatol. 1986;122:688-90. 
41. Baden HP, Kubilus J, Baden L. A stain for plucked anagen hairs. J Am Acad Dermato. 1979;1:121-2.

42. Sperling LC. Introduction to diseases of the hair. In: Demis DJ, editor. Clinical dermatology. 18th edition. New York: Lippincott; 1991. p. 1-17.

43. Caseiro RJ. Diagnostic techniques for hair disorders: III. Clinical hair manipulations and clinical findings. Cutis. 1987;40:442-8.

44. Olsen EA, Bettencourt MS, Coté NL. The presence of loose anagen hairs obtained by hair pull in the normal population. J Investig Dermatol Symp Proc. 1999;4:258-60.

45. Grover C, Nanda S, Reddy B, Chaturvedi K. Nail biopsy: Assessment of indications and outcome. Dermatol Surg. 2005;31:190-4.

46. Barrera-Vigo MV, Tejera-Vaquerizo A, Mendiola-Fernández M, Cid J, Cabra-de Luna B, Herrera-Ceballos E. Diagnostic utility of nail biopsy: A study of 15 cases. Actas Dermosifiliogr. 2008;99:621-7. Fecha de consulta: 10 de noviembre de 2013. Disponible en:http://apps.elsevier.es/watermark/ctl_servlet?_f=10\&pident_ articulo $=90183286 \&$ pident_usuario $=0 \&$ pcontactid $=\&$ pident_re vista $=403 \&$ ty $=109 \&$ accion $=$ L\&origen $=$ actasdermo\&web=http:// www.actasdermo.org\&lan=en\&fichero=403v99no8a9018328 6pdfoo1.pdf.

47. Hanno R, Mathes BM, Krull EA. Longitudinal nail biopsy in evaluation of acquired nail dystrophies. J Am Acad Dermatol 1986;14:803-9.

48. deBerker DA, Dahl MG, Comaish JS, Lawrence CM. Nail surgery: An assessment of indications and outcome. Acta Derm Venereol 1996;76:484-7.

49. Rich P. Nail biopsy. Indications and methods. J Dermatol Surg Oncol. 1992;18:673-82.

50. Rich P. Nail biopsy: Indications and methods. Dermatol Surg. 2001;27:229-34.

51. Grover C, Khandpur S, Reddy B, Chaturvedi K. Longitudinal nail biopsy: Utility in 20-nail dystrophy. Dermatol Surg. 2003;29:1125-9.

52. Martin B. Nail histopathology. Actas Dermosifiliogr. 2013;104(7):564-78

53. Haneke E. Cirugía dermatológica de la región ungular. Monogr Dermatol. 1991;6:408-11.

54. André J, Sass U, Richert B, Theunis A. Nail pathology. Clin Dermatol. 2013;31:526-39.
55. Fleckman P, Omura EF. Histopathology of the nail. Adv Dermatol. 2001;17:385-406.

56. Haneke E. Surgical anatomy of the nail apparatus. Dermatol Clin. 2006;24:291-6.

57. Weinberg JM, Koestenblatt EK, Jennings MB. Utility of histopathologic analysis in the evaluation of onychomycosis. J Am Podiatr Med Assoc 2005;95:258-63.

58. Grover C, Reddy BS, Chaturvedi KU. Onychomycosis and the diag nostic significance of nail biopsy. J Dermatol. 2003;30:116-22.

59. González-Serva A. Structure and function. In: Scher RK, DanielCR, editors. Nails: therapy, diagnosis, surgery. Philadelphia: Saunders; 1997:11-30.

6o. Ruben BS. Pigmented lesions of the nail unit: Clinical and histologic features. Semin Cutan Med Surg. 2010;29:148-58.

61. Martin B. Histopatología de la uña. Actas Dermosifiliog. 2013;104:564-78.

62. Lewin K, De Wit AS, Lawson R. Softening techniques for nails biopsies. Arch Dermatol. 1973;107:223-4.

63. Piérard GE, Arrese JE, Pierre S, Bertrand C, Corcuff P, Lévêque JL, Piérard-Franchimont C. Diagnostic microscopique des onychomycoses. Ann Dermatol Venerol. 1994;121:25-9.

64. Hay RJ, Baran R, Haneke E. Fungal (onychomycosis) and other infections involving the nail apparatus. In: Baran R, Dawbern RPR, de Berker DAR, Haneke E, Tosti A, editors. Baran and Dawbern's diseases of the nails and their management. Malden, MA: Blackwell Science; 2001:211-50.

65. Perrin C, Michiels JF, Pisani A, Ortonne JP. Anatomic distribution of melanocytes in normal nail unit: An immuno-histochemical investigation. Am J Dermatopathol. 1997;19:462-7.

66. Tosti A, Cameli N, Piraccini BM, Fanti PA, Ortonne JP. Characterization of nail matrix melanocytes with anti-PEP1, anti-PEP8, TMH-1, and HMB-45 antibodies. J Am Acad Dermatol. 1994;31:193-6.

67. Jerasutus S. Histopathology. In: Scher RK, Daniel CR 3rd, editors. Nails: Diagnosis, therapy, surgery. 3rd edition. Philadelphia: Elsevier Saunders; 2005. p. 37-70.

68. Sellheyer K, Bergfeld WF, Stewart E, Roberson G, Hammel J. Evaluation of surgical margins in melanocytic lesions: A survey among 152 dermatopathologists. J Cutan Pathol. 2005;32:293-9. 